\title{
On Derivation of the Probability of Occurrence of an Epidemic with Application to HIVIAIDS Spread Given Tuberculosis Co-infections in the Presence of Treatment
}

\author{
Richard Onyino Simwa ${ }^{1, *}$, Nelson Lwoyelo Muhati ${ }^{2}$ Lucy Chikamai ${ }^{2}$ \\ ${ }^{1}$ School of Mathematics, University of Nairobi, Nairobi, Kenya \\ ${ }^{2}$ Department of Mathematics, Kibabii University, Bungoma, Kenya
}

Email address:

rsimwaa@uonbi.ac.ke (R. O. Simwa), nellymuhati@yahoo.com (N. L. Muhati), lucychikamai@gmai.com (L. Chikamai)

${ }^{*}$ Corresponding author

\section{To cite this article:}

Richard Onyino Simwa, Nelson Lwoyelo Muhati, Lucy Chikamai. On Derivation of the Probability of Occurrence of an Epidemic with Application to HIV/AIDS Spread Given Tuberculosis Co-infections in the Presence of Treatment. Science Journal of Applied Mathematics and Statistics. Vol. 5, No. 5, 2017, pp. 169-173. doi: 10.11648/j.sjams.20170505.11

Received: July 11, 2017; Accepted: July 21, 2017; Published: August 22, 2017

\begin{abstract}
Human Immunodeficiency Virus (HIV) and Mycobacterium Tuberculosis (TB) infections are two major world's public health problems especially in developing countries. Worldwide, $13 \%$ of TB cases are estimated to be co-infected with HIV and about a third of 33 million people living with HIV are infected with the bacterium that causes TB. Deterministic models are derived and applied to estimate the basic reproduction number of HIV and TB co-infection as a single output value by treating each of the parameter input as a constant value. In this paper the basic reproduction number is modeled as a random variable, then the probability that there will bean epidemic, is derived and computed. In particular it is shown that for the subSaharan region, the probability of the epidemic occurring is $7.9 \%$, as expected since an epidemic is generally a rare event. This research, thus develops the methodology for the computation of the probability of occurrence of an epidemic, which is useful for the public health policy formulation.
\end{abstract}

Keywords: Normal Distribution, Basic Reproduction Number, Probability of an Epidemic, HIV/AID Sand TB Co-Infection Modeling, Stochastic Simulations, Ordinary Differential Equations

\section{Introduction}

HIV and TB co-infection have a global prevalence with devastating morbidity and massive mortality [1]. HIV infected individuals have a higher chance of being infected with TB due to their weak immune system resulting from depletion of $C D 4^{+}$lymphocyte. The impaired immunity results to increased number of primary TB cases and reactivation of TB in HIV infected individuals [2]. Thus, $\mathrm{HIV}$ is said to be the most powerful risk for progression from TB infection to TB disease [3]. An HIV positive person infected with Mycobacterium tuberculosis has a 50\% chance of developing active TB against a $10 \%$ chance for the HIV negative [3]. Worldwide, TB is the most common opportunistic infection affecting HIV positive individuals [4] and it remains to be the most common cause of death in patients with AIDS [5]. It enhances HIV replication leading to increased viral load by accelerating the natural evolution of HIV infection [6] hence increasing the risk of progression from HIV to AIDS ([7], [8]). In 2013, about 9.0 million people had developed TB while 1.5 million died from the disease with 360000 being HIV-positive [9]. Although deterministic models have been developed and analyzed to estimate the basic reproduction number for HIV and TB coinfection ([10], [11], [12]), not much has been done to estimate the basic reproduction number as a random variable using probabilistic approach. Probabilistic modeling is a statistical analysis tool that estimates on the basis of past (historical) data the probability of an event occurring again. It differs from a deterministic approach because one can change the values of parameter input assumptions at random in order to see the impact of these changes on the output. This approach make use of Monte Carlo simulations to stimulate the variation in the basic reproduction number, by running a 
deterministic model several thousands times. Different studies ([13], [14] and [15]) have used this approach to estimate the basic reproduction number as a random variable. In this paper extents these results to determine the probability of an epidemic, and show that in this case the probability of occurrence of the epidemic is $7.9 \%$, which lies within the expected public health limits for an epidemic.

\section{Methodology}

The probability of an epidemic is derived, in this section, first by determining the expression for the basic reproduction number in terms of the parameters, in particular with respect to the HIV/AIDS pandemic given TB co-infections in the presence of treatment application. This is achieved in section 2.1. The probability of an epidemic is then derived and applied in section 2.2.

\subsection{The Dynamical HIV/AIDS Model Given TB Co-infections with Treatment Application}

The total population is divided into the following subgroups of susceptible individuals

(S), those infected with HIV only $\left(I_{H}\right)$, individuals infected with TB only $\left(I_{T}\right)$, HIV individuals dually infected with TB $\left(I_{H T}\right)$, those treated of HIV and TB (T) and AIDS individuals (A).

Thus the total population at time $\mathrm{t}$ is given as;

$$
N(t)=S(t)+I_{H}(t)+I_{T}(t)+I_{H T}(t)+T(t)+A(t)
$$

The compartmental diagram in Figure 1 illustrates the flow of individuals as they face the possibility of acquiring specific infections or co-infection. It is assumed that susceptible individuals are recruited into the population at a constant rate of $\Lambda$. Susceptible individuals acquire HIV infection following effective contact with HIV infected individuals at a rate $\lambda_{H}$ which is given as;

$$
\lambda_{\mathrm{H}}=\beta_{\mathrm{H}} \frac{\left(I_{H}+\theta I_{H T}\right)}{N}
$$

where $\beta_{H}$ is the probability of getting infected from a randomly chosen partner and $\theta$ is a modification parameter which accounts for the relative infectiousness of dually infected individuals.

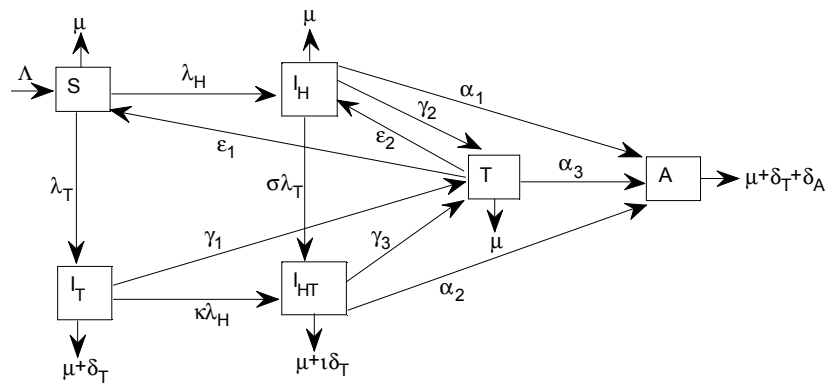

Figure 1. Model flow diagram for HIV and TB Co-infection.

Similarly, susceptible individuals acquire TB infection following contact with TB infectious individual at a rate $\lambda_{T}$ given as;

$$
\lambda_{\mathrm{T}}=\beta_{\mathrm{T}} \mathrm{C} \frac{\left(I_{T}+\eta I_{H T}\right)}{N}
$$

where $\beta_{T}$ is the probability of being infected with one infectious individual, $\mathrm{C}$ is contact rate and $\eta$ is a modification parameter which accounts for the relative infectiousness of dually infected individuals.

It is assumed that the probability of survival till infection state for individuals infected with TB is unity i.e. excludes exposed class and that TB infected individuals may recover after treatment and return to the susceptible class at a rate of $\epsilon_{1}$. All individuals suffer from natural death in different subpopulation at a constant rate of $\mu$. The HIV and TB model is given by the following systems of six Ordinary Differential Equations (ODEs):

$$
\left.\begin{array}{c}
\frac{d S}{d t}=\Lambda+\epsilon_{1} T-\left(\lambda_{H}+\lambda_{T}+\mu\right) S \\
\frac{d I_{H}}{d t}=\lambda_{H} S+\epsilon_{2} T-\sigma \lambda_{T} I_{H}-\left(\alpha_{1}+\gamma_{2}+\mu\right) I_{H} \\
\frac{d I_{H}}{d t}=\lambda_{T} S-\kappa \lambda_{H} I_{T}-\left(\mu+\delta_{T}+\gamma_{1}\right) I_{T} \\
\frac{d I_{H T}}{d t}=\sigma \lambda_{T} I_{H}+\kappa \lambda_{H} I_{T}-\left(\alpha_{2}+\gamma_{3}+\iota \delta_{T}+\mu\right) I_{H T} \\
\frac{d T}{d t}=\gamma_{2} I_{H}+\gamma_{1} I_{T}+\gamma_{3} I_{H T}-\left(\epsilon_{1}+\epsilon_{2}+\alpha_{3}+\mu\right) T \\
\frac{d A}{d t}=\alpha_{1} I_{H}+\alpha_{2} I_{H T}+\alpha_{3} T-\left(\mu+\delta_{T}+\delta_{A}\right) A
\end{array}\right\}
$$

Table 1. Definitions of Model Parameters used in Equation (4).

\begin{tabular}{ll}
\hline Parameter & Description \\
\hline$\Lambda$ & Recruitment rate \\
$M$ & Natural mortality \\
$\lambda_{T}$ & Force of infection due to TB \\
$\lambda_{H}$ & Force of infection due to HIV \\
$\gamma_{1}$ & Rate at which TB infected individuals progress for treatment \\
$\gamma_{2}$ & Rate at which HIV infected individuals progress for treatment \\
$\gamma_{3}$ & Rate at which dually-infected individuals progress for treatment \\
$\sigma$ & Modification parameter for increased susceptibility to TB infection as a result of HIV infection \\
$\kappa$ & Modification parameter for increased susceptibility to HIV infection as a result of TB infection \\
$\iota$ & Modification parameter for increased TB death rate as a result of HIV infection \\
$\epsilon_{1}$ & Rate at which TB treated individuals goes back to susceptible class \\
$\epsilon_{2}$ & Rate at which HIV treated individuals goes back to HIV class \\
$\delta_{T}$ & TB related death rate \\
$\delta_{A}$ & AIDS related death rate \\
\hline
\end{tabular}




\begin{tabular}{ll}
\hline Parameter & Description \\
\hline$\alpha_{1}$ & Rate at which HIV individuals progress to full blown AIDS \\
$\alpha_{2}$ & Rate at which dually-infected individuals progress to full blown AIDS \\
$\alpha_{3}$ & Rate at which dually-infected treated individuals progress to full blown AIDS \\
\hline
\end{tabular}

\subsubsection{Positivity and Boundedness of Solutions}

Since the model represented by system (4) is dealing with human population, all state variables and parameters are assumed to be non-negative for all $t \geq 0$ hence initial conditions are given by;

$$
\begin{gathered}
S(0)=S_{0} \geq 0, I_{H}(0)=I_{H 0} \geq 0, I_{T}(0)=I_{T 0} \geq 0, I_{H T}(0)=I_{H T 0} \geq 0, \\
T(0)=T_{0} \geq 0, A(0)=A_{0} \geq 0, N(0)=N_{0} \geq 0
\end{gathered}
$$
(1).

The boundedness of solution can be proved using equation since;

$$
0 \leq N(t)=S(t)+I_{H}(t)+I_{T}(t)+I_{H T}(t)+T(t)+A(t) \leq \frac{\Lambda}{\mu}
$$

Then, any variable of $\left[S(t), I_{H}(t), I_{T}(t), I_{H T}(t), T(t), A(t)\right]$ lies between $\left[0, \frac{\Lambda}{\mu}\right]$

Hence the model represented by system (4) is studied in a suitable feasible region;

$$
\Psi=\left[\left(\mathrm{S}, \mathrm{I}_{\mathrm{H}}, \mathrm{I}_{\mathrm{T}}, \mathrm{I}_{\mathrm{HT}}, \mathrm{T}, \mathrm{A}\right) \in R_{+}^{6}: \mathrm{N}(\mathrm{t}) \leq \frac{\Lambda}{\mu}\right]
$$

\subsubsection{Basic Reproduction Number $R_{h t}$ for HIV and TB Co-Infection}

The basic reproduction number $R_{h t}$ for HIV and TB coinfection is defined as the number of secondary HIV and TB infections due to a single HIV and TB infective individual. We use the next matrix generation operator approach (see [16]) to arrive at an expression for $R_{h t}$ as;

$$
\mathrm{R}_{\mathrm{ht}}=\max \left(\frac{\beta_{H}}{\alpha_{1}+\gamma_{2}+\mu}, \frac{\beta_{T} C}{\delta_{T}+\gamma_{1}+\mu}\right)
$$

\subsubsection{Local Stability of Disease-Free Equilibrium}

Corollary 1 follows from Theorem 2 in [16].

Corollary 1 The Disease free Equilibrium of the system (DFE) in Equation (4) is locally asymptotically stable if $R_{h t}<1$ and unstable if $R_{h t}>1$.

Corollary 1 can be proven using the Jacobian matrix of Equation (4) at DFE.

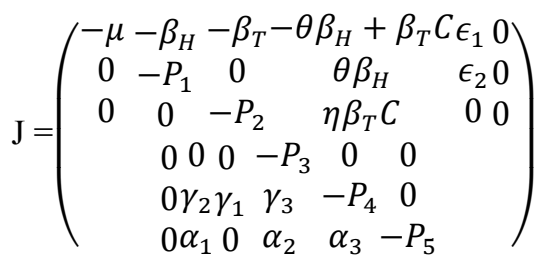

where $P_{1}=\beta_{H^{-}}\left(\alpha_{1}+\gamma_{2}+\mu\right), \quad P_{2}=\beta_{T} C-\left(\mu+\delta_{T}+\gamma_{1}\right), \quad P_{3}=$ $\left(\alpha_{2}+\gamma_{3}+l \delta_{T}+\mu\right), P_{4}=\left(\epsilon_{1}+\epsilon_{2}+\alpha_{3}+\mu\right), P_{5}=\left(\mu+\delta_{T}+\delta_{A}\right)$

Using Mathematica, the eigenvalues for Equation (6) at $\mathrm{DFE}$ are given as;

$$
\lambda_{\mathrm{i}}=-\mu, \beta_{\mathrm{H}^{-}}\left(\alpha_{1}+\gamma_{2}+\mu\right), \beta_{\mathrm{T}} \mathrm{C}-\left(\mu+\delta_{\mathrm{T}}+\gamma_{1}\right),-\left(\alpha_{2}+\gamma_{3}+1 \delta_{\mathrm{T}}+\mu\right),-
$$

$$
\left(\epsilon_{1}+\epsilon_{2}+\alpha_{3}+\mu\right) \text { and }-\left(\mu+\delta_{\mathrm{T}}+\delta_{\mathrm{A}}\right)
$$

For asymptotic stability, all the eigenvalues at DFE should be negative.

Therefore;

$$
\mathrm{R}_{\mathrm{h}}=\frac{\beta_{H}}{\alpha_{1}+\gamma_{2}+\mu}<1, \mathrm{R}_{\mathrm{t}}=\frac{\beta_{T} C}{\delta_{T}+\gamma_{1}+\mu}<1
$$

Where $R_{h}$ and $R_{t}$ is the reproduction number for HIV and TB respectively.

It can be noted that the DFE is locally asymptotically stable if $R_{h}<1$ and $R_{t}<1$ thus the disease dies out. Hence at DFE, the basic reproduction number $R_{h t}$ is locally asymptotically stable if $R_{h t}<1$ and unstable if either of $R_{h}>1$ or $R_{t}>1$.

\subsection{Probability Distribution of the Basic Reproduction Number}

In this section we determine the probability distribution of the basic reproduction number $R_{h t}$ for HIV and TB coinfection with random effect in parameters.

\subsubsection{Distributions of Model Parameters}

The basic reproduction number $R_{h t}$ for HIV and TB coinfection given in Equation (5) is a function of eight different parameters which yields a single output when a single value of parameter is used. This study considers the parameters of the model to be random variables following different probability distribution because each of the model's parameters is a random variable. The distribution of the basic reproduction number is then studied with the random effects in the parameters. A probability density function is assigned to each parameter, based on their range of possible values and the probability of occurrence of any specific values. The possible values and the distribution of the parameters areas given in [15], see Table 2.

Table 2. Parameter Estimators and their Distributions.

\begin{tabular}{llllll}
\hline Parameter & Units & Minimum & Peak & Maximum & Reference \\
\hline$M$ & Per year & 0.02 & & 0.025 & {$[17],[18]$} \\
$\beta_{T}$ & Per year & 0.1 & 0.35 & 0.6 & {$[19]$} \\
$\beta_{H}$ & Per year & 0.011 & 0.075 & 0.095 & {$[20]$} \\
$\delta_{T}$ & Per year & 0.01 & & 0.02 & {$[19]$} \\
$\gamma_{1}$ & Per year & 0.55 & & 0.60 & Uniform \\
$\gamma_{2}$ & Per year & 0.7 & & 0.75 & Uniform \\
$\alpha_{1}$ & Per year & 0.1 & & 0.16 & Uniform \\
$\mathrm{C}$ & Per year & 3.0 & & 3.2 & Uniform \\
\hline
\end{tabular}

Two of the eight parameters are assumed to follow a triangular probability distribution ([21], [22]). The selection of this distribution is preferred because the peak value and range (difference between the minimum and maximum) since the parameters are estimable [22]. On the other hand, the remaining six parameters are assumed to follow a uniform 
distribution (labeled 'uniform' in Table 2) because only the range of the parameter is estimable (see [22]).

\subsubsection{Simulations for the Distribution of the Basic Reproduction Number}

Markov Chain Monte Carlo (MCMC) simulation approach is one of the most appropriate sampling scheme resulting into $\mathrm{N}=1,000$ samples for each parameter. The basic reproduction number $R_{h t}$ is then computed and its empirical probability value calculated from a sample of 35,000 iterations for each parameter when its value is less than one and when greater than one. MCMC approach in Win BUGS is used to generate data which is then imported to R software in order to fit the probability distribution of $R_{h t}$.

\section{Results and Discussions}

The empirical distribution of $R_{h t}$ determined using the approach in section 2.2.2 is analysed, the main results are as given in Figures 2, 3 and 4.

\subsection{Histogram and Probability Distribution of $\boldsymbol{R}_{h t}$}

The histogram and probability distributions for $R_{h t}$ are as shown in the Figures 2 to 4 . The fitted probability distribution for histogram in Figure 2 is an approximation of the observed data whose parameters are given in Table 2 and their Q-Q plot illustrated in Figure 4.

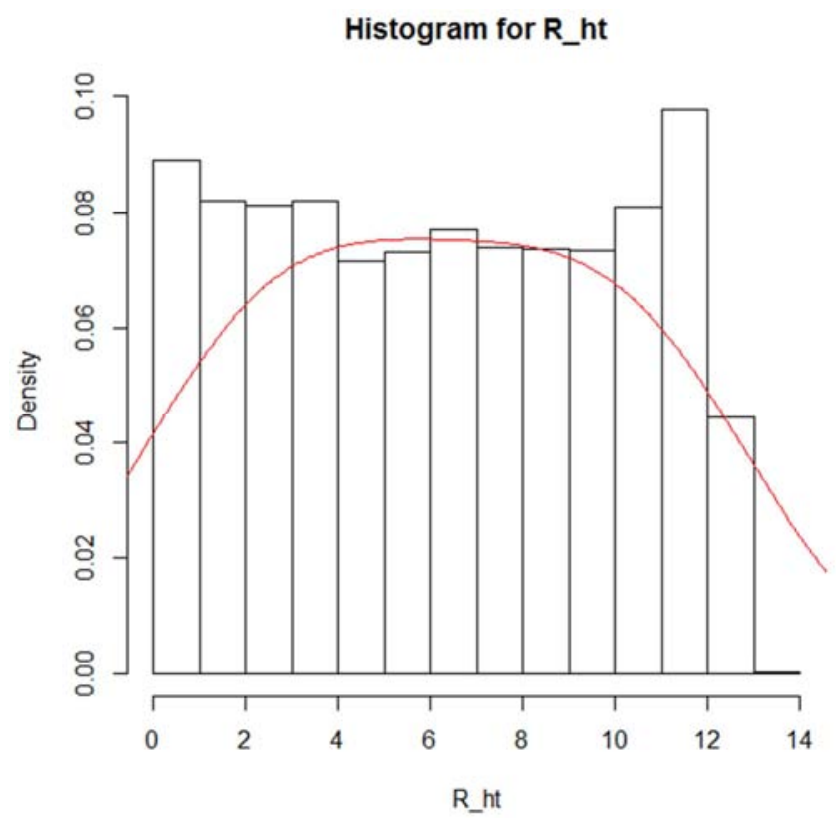

Figure 2. Histogram with normal curve showing parameter estimate for $R_{h t}$.

The normal curve in Figure 2 shows that the initial stage of transmission of the HIV and TB co-infection increases sharply towards the right hand side of the distribution due to misdiagnosis or contacts with infected object or person with HIV. This is followed by a decrease in transmission towards the right hand tail of the distribution due to individuals accessing treatment or control measures being undertaken by the public health officers for example abstaining, being faithful to one uninfected person and using condoms (ABC).

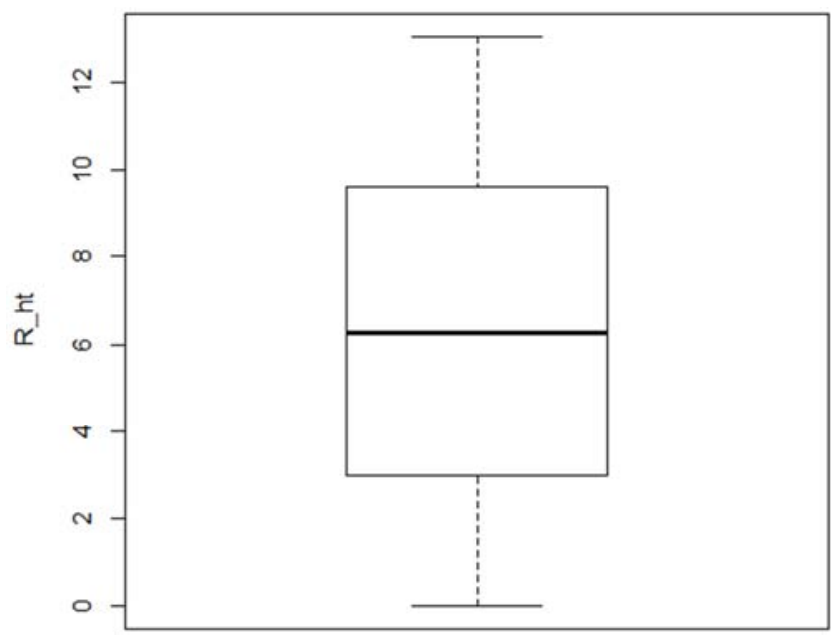

Figure 3. Box plot showing parameter estimate for $R_{h t}$.

From the box plot, it is observed that the distribution of $R_{h t}$ appears to be symmetric and approximately normal.

Q-Q plot for R_ht

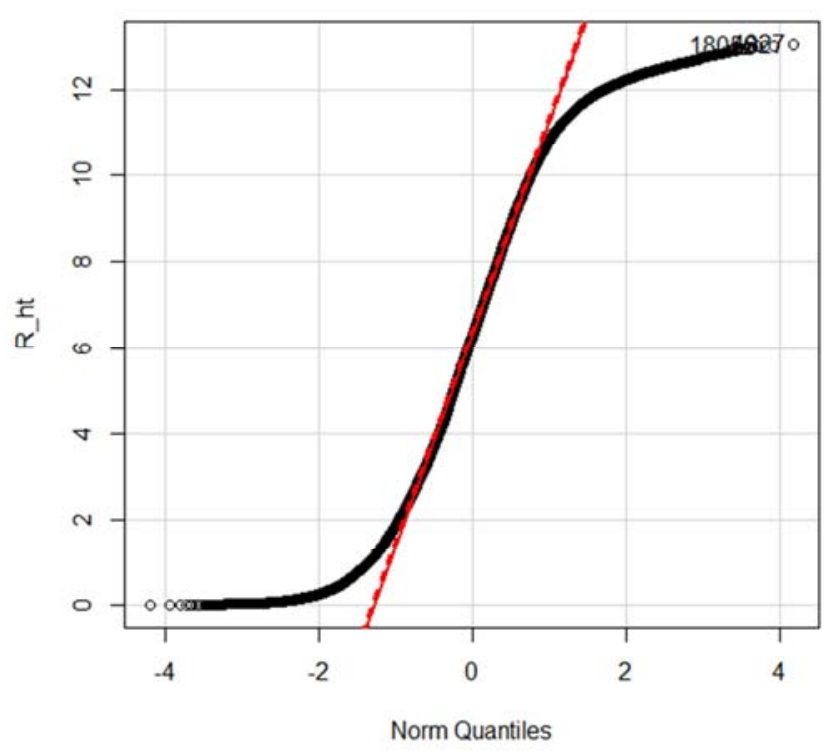

Figure 4. Normal quantile plot showing parameter estimate for $R_{h t}$.

In the above plot, the points fall very close to the straight line hence the data is approximately normally distributed with mean 6.28 and standard deviation 3.12.

The 1- sample Kolmogorov-smirnov test is used to test the goodness-of-fit of the empirical distribution for $R_{h t}$ to the normal distribution with mean 6.28 and standard deviation 3.741. The result from a sample data of 35,000 shows that the $\mathrm{P}$-value of the test is 0.4603 . Since the $\mathrm{P}$-value is greater than 0.05 , the null hypothesis is not rejected and hence there is no evidence against the observation that the distribution of basic reproduction number $R_{h t}$ does not differ significantly from the normal distribution, with mean 6.28 and standard deviation 3.741, symbolically, 


$$
R_{h t} \sim \aleph\left[6.28,3.741^{2}\right]
$$

\subsection{Probability of an Epidemic}

For any epidemic, once the distribution of the disease reproduction rate, say $\mathrm{R}$, is determined, the probability of an epidemic is then given by

$$
\text { Prob. }(R>1)=1-\mathrm{F}_{\mathrm{R}}(1)
$$

Where $F_{R}(x)$ is the cumulative distribution of the random variable $\mathrm{R}$.

Thus from the expression in (8) and Equation (9), for the HIV/IDS spread given TB co-infections with treatment application, the probability of the epidemics given by

$$
\text { Prob. }\left(\mathrm{R}_{\mathrm{ht}}>1\right)=\text { Prob. }(\aleph[6.28,3.7412]>1]=0.079=7.9 \%(10)
$$

Probability of $7.9 \%$ corresponds to the occurrence of a rare event, and this concurs with what is expected of epidemics in general.

\section{Conclusion}

This study gives the derivation of the distribution for the basic reproduction number and applies the result to compute the probability of occurrence of an epidemic, in particular the probability of an epidemic with respect to the HIV/AIDS given TB co-infection with treatment application. The probability of the epidemic occurring is found to be equal to $7.9 \%$, which is within the expected range from public health consideration, that an epidemic is a rare occurrence.

\section{References}

[1] WHO (2013). Global tuberculosis report 2013.

[2] Friedl and G, Churchyard GJ and Nardell E. (2007). Tuberculosis and HIV coinfection: current state of knowledge and research priorities. J Infect Dis 1 (9-6).

[3] Naresh R., Tripathi A., Omar S., (2006). Modelling the spread of AIDS epidemic with vertical transmission. Applied Mathematics and Computation.

[4] Adewale S. O., Olopade I. A., Adeniran G. A. and Ajao S. O. (2015). Mathematical modelling and sensitivity analysis of HIV-TB co-infection. Journal of advances in Mathematics vol. 11 , no. 8 .

[5] Raviglione MD, Snider DE, Kochi A, (1995). Global epidemiology of tuberculosis: morbidity and mortality of a worldwide epidemic. Washington D. C.

[6] Rosas-Taraco A. G, Arce-Mendoza A. Y, Caballero-Olin G, and Salinas-Carmona M. C. (2006). Mycobacterium tuberculosis upregulates coreceptors CCR5 and CXCR4 while HIV modulates CD14 favoring concurrent infection. AIDS Res Hum Retroviruses 22(1):45-51.

[7] AIDS control and prevention (AIDSCAP) (1996). The status and Trends of the global HIV/AID Spandemic.
[8] Sharma S. K, Mohan A, Kadhiravan T. (2005). HIV-TB coinfection: epidemiology, diagnosis and management. Indian $\mathrm{J}$ Med Res 121(4):550-567.

[9] WHO (2015). Global Tuberculosis Report. http://www.who.int/tb/areas-ofwork/tb-hiv/en/

[10] Adewale S. O., Olopade I. A., Adeniran G. A. and Ajao S. O. (2015). Mathematical modelling and sensitivity analysis of HIV-TB co-infection. Journal of advances in Mathematics vol. 11 , no. 8 .

[11] AIDS control and prevention (AIDSCAP) (1996). The status and Trends of the global HIV/AID Spandemic.

[12] Fatmawati and Hengki T. An Optimal Treatment Control of TB/HIV Co-infection (2016). International Journal of Mathematics and Mathematical Sciences. Volume 2016, ID8261208 (OR http://dx.doi.org/10.1155/2016/8261208).

[13] Johnson N. L, Kotz S. (1970). Distributions in statistics: continuous univariate distributions. Vol 2. New York, NY: John Wiley and Sons, Inc.

[14] Ong'ala J. O, Mugisha J. and Oleche P. (2014). A Probabilistic Estimation of the Basic Reproduction Number: A Case of Control Strategy of Pneumonia. Science Journal of Applied Mathematics and Statistics 2(2): 53-59.

[15] Sanchez M. and Blower S. (1997). Uncertainty and Sensitivity Analysis of the Basic Reproductive Rate: Tuberculosis as an Example. American Journal of Epidemiology. Vol. 12(145) pp. 1127-1137.

[16] Van den Driesche P. and Watmough J. (2005). Reproduction numbers andsub-threshold endemic equilibria for the compartmental models of disease transmission. Math Biosci 180: 29-48.

[17] Dye C., Garnett, G. P., Sleeman, K. and Williams, B. G. (1998). Prospects for worldwide tuberculosis control under the WHO DOTS strategy. Directly observed short-course therapy. Lancet.

[18] Dye C., Garnett, G. P., Sleeman, K. and Williams, B. G. (1998). Prospects for worldwide tuberculosis control under the WHO DOTS strategy. Directly observed short-course therapy. Lancet.

[19] Diekmann O., Heesterbeek, J. A. P., Metz, J. A. P., (1990). On the definition and computation of the basic reproduction ratio Ro in models for infectious diseases in heterogeneous populations. J Math Biol 365-382.

[20] Hoffmann C. (2007). Opportunistic Infections: In Bernd Sebastian Kamps, ed. HIV Medicine. 15th Edition. Flying Publisher, Paris, p. 389-477.

[21] Hyman J. M., Li J. and Stanley, E. A. (1999). The differential infectivity and staged progression models for the transmission of HIV. Math Biosci 155(2): 77-109.

[22] Swartzman G. L and Kaluzny S. P. (1987). Ecological simulation primer. New York, NY: Macmillan Publishing Company. 